\title{
Yolk sac-embryo distance in correlation with soluble form of vascular endothelial growth factor levels in pregnancy with potentially reserved evolutivity
}

\author{
Carmen Elena Bucuri' ${ }^{1}$, Razvan Ciortea ${ }^{1}$, Doru Diculescu ${ }^{1}$, Andrei Malutan', \\ Radu Mocan-Hognogi ${ }^{1}$, Costin Berceanu ${ }^{2}$, Renata Nicula ${ }^{1}$, Maria Rada ${ }^{1}$, Dan Mihu ${ }^{1}$
}

${ }^{1} 2^{\text {nd }}$ Department of Obstetrics and Gynaecology, University of Medicine and Pharmacy "Iuliu Hatieganu", Cluj-Napoca, ${ }^{2}$ Department of Obstetrics and Gynaecology, University of Medicine and Pharmacy Craiova, Romania

\begin{abstract}
Aims: Embryonic demise is a frequent complication of the first trimester pregnancy. The purpose of this study was to evaluate the correlation between a serum biomarker, the soluble form of the vasculo-endothelial growth factor (sFlt-1) and the distance between the yolk sac (YS) and embryo (DYSE), determined by ultrasonography. Material and methods: The study was a prospective case-control study that included 2 groups of patients - the control group with 81 first-trimester pregnancies in evolution and the case group with 89 first-trimester pregnancies with a potentially reserved evolutivity. Results: A correlation between the serum level of sFlt-1 and DYSE in embryos with crown-rump length (CRL) greater than $5 \mathrm{~mm}$ was identified, showing that a DYSE $\leq 3 \mathrm{~mm}$ correlates with a low level of sFlt-1 $(\mathrm{p}<0.05)$ and a DYSE $>4$ mm correlates with an increased level of sFlt-1 $(\mathrm{p}<0.05)$. Conclusions: A low level of sFlt-1 associated with a distance between the embryo and yolk sac of small dimensions, respectively $<3 \mathrm{~mm}$, correlates with an increased rate of non-viable embryos. This correlation between an ultrasound and a serum parameter is of great value and brings important information about the viability of first trimester pregnancies.
\end{abstract}

Keywords: embryonic demise; vascular endothelial growth factor; distance yolk sac-embryo

\section{Introduction}

Embryonic demise is an entity with an increased incidence, with more than $25 \%$ of the 21 'st century female population being affected by this pathology [1]. In more than $40 \%$ of cases, the etiology of embryonic demise is the presence of chromosomal abnormalities [2].

Ultrasonography (US) is the preferred imaging technique in the diagnosis of embryonic demise [3]. The distance between the yolk sac (YS) and the embryo (DYSE) is an ultrasonographic parameter known to be useful in

Received 20.03.2018 Accepted 11.07.2018

Med Ultrason

2018, Vol. 20, No 4, 487-492

Corresponding author: Ciortea Razvan

55, 21st December Bld, Cluj-Napoca, Romania Phone: +40748030897

E-mail: r_ciortea@yahoo.com assessing the viability of the first trimester pregnancy [4]. A value of this distance of less than $3 \mathrm{~mm}$ directly correlates with the unfavorable evolution of the pregnancy. Correlation of this distance with serum levels of angiogenic markers may provide additional information on a pregnancy prognosis [5]. This finding has potential benefits for women with a prior miscarriage, aware of the fact that an early knowledge of the viability of the pregnancy would have a lower negative emotional impact [6].

The first stages of embryonic development take place in a low oxygen environment. This early physiological hypoxia of the gestational sac protects the developing embryo against the teratogenic effects of free oxygen radicals [7]. A stable oxygen gradient between maternal decidua and placental tissue is also an important factor in the differentiation and migration of trophoblasts, as well as in the normal development of vilitis and angiogenesis [8]. 
In normal pregnancies, there is a physiological oxidative stress in placental tissue at approximately 9-10 weeks of pregnancy, a phenomenon evidenced by an increase in serum level of the soluble form of vasculoendothelial growth factor (VEGF)(sFlt-1). As judged by adult tissue criteria, the human embryo develops in a low oxygen environment. The trophoblastic cells are extremely sensitive to oxidative stress because of their extensive cell divisions and the concomitant exposure of their DNA [3]. This phenomenon results in an increase in sFlt-1 production, which in turn can lead to a blockage of trophoblastic invasion [9].

The identification of a low serum level of sFlt- 1 before the embryonic demise suggests an inappropriate vascularization in the intervillary space and is associated with metabolic changes at the level of the implantation zone [10]. These changes consist of an increase in the oxygen level, knowing that the serum level of sFlt-1 is inversely correlated with the amount of oxygen [11]. Identifying a DYSE $\leq 3 \mathrm{~mm}$ in association with a low level of sFlt-1 could lead to more appropriate and individualized management.

The purpose of this study is to evaluate the correlation between a serum biomarker (sFlt-1) and an ultrasonographic parameter (DYSE) in assessing the prognosis of pregnancy evolutivity in the first trimester.

\section{Material and methods}

This study is a prospective case-control study that comprised 170 patients in the first trimester of pregnancy without a known associated pathology, a study conducted in "Dominic Stanca" Obstetrics-Gynecology Clinic between 2015-2017.

Patients were divided in 2 groups - the control group with 81 first-trimester pregnancies with DYSE $>4$ $\mathrm{mm}$ and the case group with 95 pregnant patients with DYSE $\leq 3 \mathrm{~mm}$, both groups having amenorrhea for 6-11 weeks. In terms of the number of patients included in the study of each group, homogeneous lots were made in each amenorrhea week. For 6 patients of the case group, the prediction of the studied ultrasonographic parameter to an unfavorable evolution was not confirmed by repeated examinations, being therefore excluded from the study. Finally, the control group was formed by 89 patients.

US examination was performed with a $6.5 \mathrm{MHz}$ nominal frequency endovaginal probe by the same examiner with 10 years experience in ultrasonography, using a Toshiba Aplio 300 machine. The patients were placed in dorsal decubitus with hips flexed on the abdomen at a $100^{\circ}$ angle, shoulder-width abducted and the calves

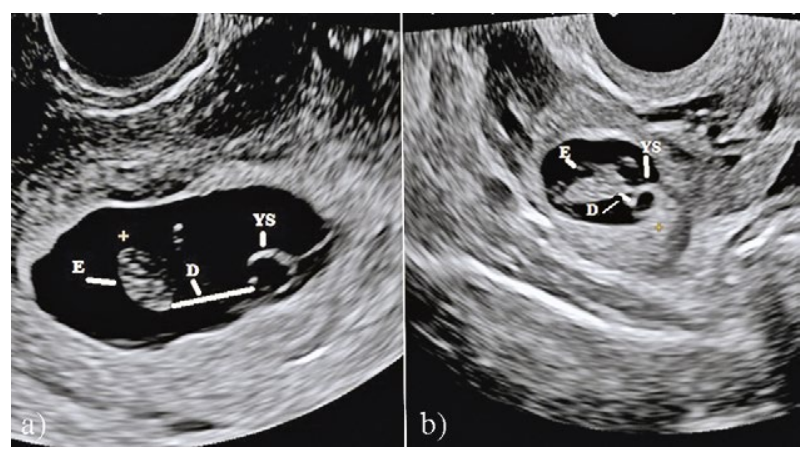

Fig 1. Embryo identified with endovaginal sonography. The embryo is recognized as a small linear echogenic structure (E) near the edge of the yolk sac (YS). The distance between the yolk sac and the embryo is outlined by a straight line (D). In the first image (a) the embryo has cardiac activity and is situated at $6 \mathrm{~mm}$ distance from the yolk sac, from a 10 weeks +1 day pregnancy and in the second image (b) there is no cardiac activity, the embryo is situated at $2 \mathrm{~mm}$ distance from the yolk sac, from a 9 weeks +1 day pregnancy. Note also that a part of the amnion is visible as a membrane encircling the embryo.

flexed on the hips at a $45^{\circ}$ angle. In order to evaluate DYSE, it was also necessary to separately evaluate the parameters included: crown-rump length (CRL) and appearance and size of YS. There were on average 2-3 serial examinations, performed at regular intervals of 3-5 days, by the same examiner, until the final diagnosis of embryonic demise. The measurements of DYSE were performed using the sagittal section and evaluating 3 different distances from the inferior pole of the embryo and YS, the smallest of the 3 distances being considered for analysis (fig 1). Only the first ultrasound examination was used to measure the DYSE, the others were designed to detect the viability of the embryo. The patients needed a different number of examinations until confirmation of the absence of embryonic cardiac activity.

During their first visit, from each subject enrolled in the study, $20 \mathrm{ml}$ of blood were collected by venipuncture into anticoagulant-free tubes for sFlt-1 dosing. In order to obtain the data we used the Human sVEGFR1/sFLT1 PicoKine ${ }^{\mathrm{TM}}$ ELISA Kit, with range of detection between $156 \mathrm{pg} / \mathrm{ml}$ and $10000 \mathrm{pg} / \mathrm{ml}$, with a sensitivity of less than $30 \mathrm{pg} / \mathrm{ml}$.

Patients' enrolment in study was made only after obtaining informed consent. The working methodology was approved by the Ethics Committee of the University of Medicine and Pharmacy "Iuliu Hatieganu" Cluj-Napoca.

\section{Statistical analysis}

Descriptive statistics elements were calculated, data presented using centrality, location and distribution in- 
dicators. The Shapiro-Wilk test was used to test normal distribution. The variance was tested using the F test. In the case of the normal distribution data, the Student $(\mathrm{t})$ test was used and in the case of non-uniform distribution values or ranks, the non-parametric Mann-Whitney (U) test was used for two non-paired samples. For the analysis of three or more samples the ANOVA test was used for normal distribution data or Kruskal-Wallis nonparametric test for non-uniform values or ranges. The significance threshold for the tests used was $\alpha=0.05$ (5\%), 0.01 $(1 \%)$ and 0.001 respectively. The Pearson correlation coefficient (r) was used to detect the correlation between two continuous quantitative variables with normal distribution. In the case of non-uniform variables, Spearman ( $\rho$ ) correlation coefficient was used. Analysis of correlation coefficients was performed using Colton's rule. The method used to obtain the mathematical equation of the dependence of a continuous variable to another variable was the linear regression. The equation of a line is $y=a+$ $b x$, where a, the intercept, is the value of " $y$ " when " $x$ " is equal to zero and $b$ (coefficient of regression) is the number for the gradient or slope of the straight line (regression slope). $\mathrm{R}^{2}$, the coefficient of determination, is the proportion of the variance in the dependent variable that is predictable from the independent variable. In linear regression $\mathrm{R}^{2}$ is the square of the correlation coefficient.

\section{Results}

No statistically significant difference between the two groups was observed regarding the gestational age (GA) $(8.49 \pm 1.59 \mathrm{~mm}$ for control group and $8.56 \pm 1.62$ case group, $p>0.05$ ) or CRL value but a statistically significant difference was obtained when DYSE values were compared $(\mathrm{p}<0.001)$ (table I, fig 2).

sFlt-1 levels were significantly higher in the control group (values between $1066.91-3961.44 \mathrm{pg} / \mathrm{ml}$ ) compared with the case group (values between 568.7 $990.55 \mathrm{pgml})(\mathrm{p}<0.001)($ fig 3$)$.

A DYSE $\leq 3 \mathrm{~mm}$, considered an abnormal distance at gestational age $\geq 6$ weeks of amenorrhea, correlates with

Table I. Ultrasonographic indicators: comparative analysis and statistical significance

\begin{tabular}{llll}
\hline Parameter & Group & Mean \pm SD (Min-Max) & p \\
\hline DYSE (mm) & I (n=81) & $5.57 \pm 1.33(3-9)$ & $<0.0001$ \\
& II (n=89) & $2.6 \pm 1.43(1-8)$ & \\
CRL (mm) & I (n=81) & $20.8 \pm 1.10(6-43)$ & 0.49 \\
& II (n=89) & $19.5 \pm 1.01(6-42)$ & \\
\hline
\end{tabular}

SD - standard deviation; $n$ - number of patients; DYSE - distance between yolk sac and embryo; CRL - crown-rump length

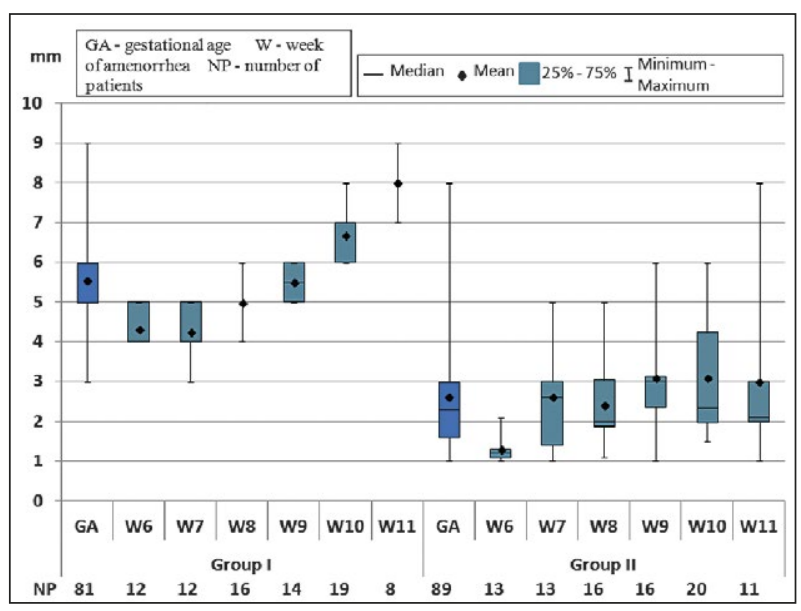

Fig 2. Box plots of the distance between yolk sac and embryo (DYSE) in the normal and case groups, according to the gestational age.

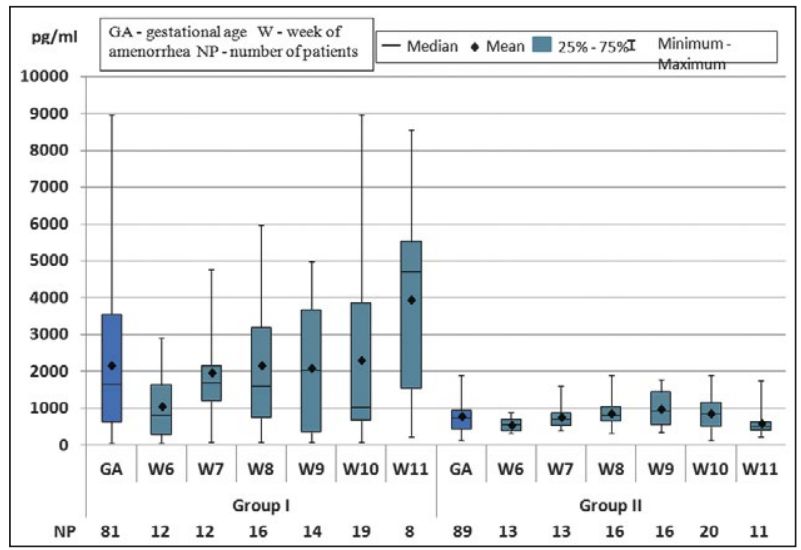

Fig 3. Box plots of soluble fms-like tyrosine kinase 1 (sFlt-1) in the normal and case groups, according to the gestational age.

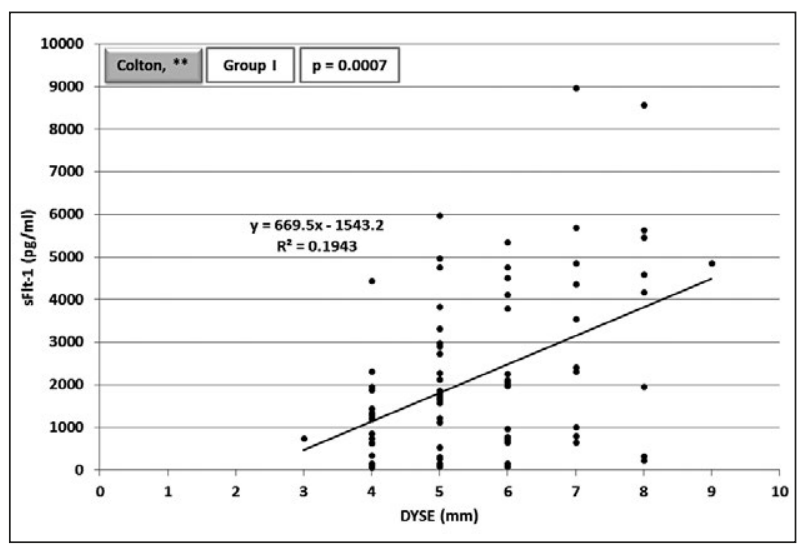

Fig 4. The correlation between soluble fms-like tyrosine kinase 1 (sFlt-1) and yolk sac-embryo distance (DYSE) in the normal group 


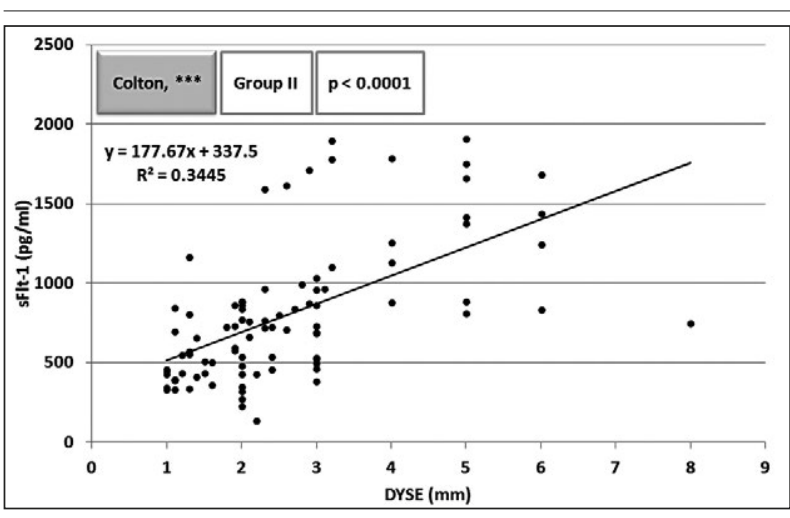

Fig 5. The correlation between soluble fms-like tyrosine kinase 1 (sFlt-1) and yolk sac-embryo distance (DYSE) in the case group

a low value of sFlt- $1(\mathrm{p}<0.05)$ and inversely, a DYSE $>4$ $\mathrm{mm}$, considered normal at this gestational age, correlates with an increased value of sFlt-1( $\mathrm{p}<0.05$ ) (fig 4 , fig 5).

\section{Discussions}

In this study we showed a correlation between the serum level of sFlt-1 and the unfavorable evolution of the first trimester pregnancy but also that a DYSE value of less than $3 \mathrm{~mm}$ correlates with a low level of sFlt-1. Although the sFlt-1 role in the first-trimester pregnancy pathology is known [12], to our knowledge, this is the first study in the literature to demonstrate the correlation between an ultrasound parameter (DYSE) and this serum parameter.

In its early development, the embryo is located immediately adjacent to YS. Separation of the YS from the embryo is due to the development of the embryonic structure. As the embryo develops, DYSE increases, going beyond $3 \mathrm{~mm}$. For embryos with $\mathrm{CRL} \leq 5 \mathrm{~mm}$, there should be no separation of YS [13]. However, there is a study that demonstrated that in some pregnancies with small embryos, there is an unexpected separation of the YS from the embryo [10]. We found this situation in the 6 patients excluded from the study. In most cases when the embryo reaches a CRL $>5 \mathrm{~mm}$, there is a clear separation of the YS. When this separation is observed, the embryo's cardiac activity should be invariably detected. The absence of this separation could be used as a marker in the diagnosis of pregnancy with potentially reserved evolutivity [14]. Filly et al argue that this ultrasonographic parameter, respectively DYSE, is valuable in anticipating an unfavorable pregnancy evolutivity, whether used alone or in combination with other abnormal features [15].

From the point of view of ultrasonography, the pregnancy with potentially reserved evolutivity is still an in- sufficiently explored topic and therefore there is a great need for prospective data on prognostic factors that could be useful in establishing a high accuracy prognosis. So, transvaginal ultrasonography has become an important method of assessing the first trimester pregnancy and is helping to identify new parameters of evolutivity [16]. The ultrasonographic diagnosis of a pregnancy with potentially reserved evolutivity is based on the quantification of specific ultrasound parameters: absence of YS or increase in size over $6 \mathrm{~mm}$, YS deficiency of vascularization, embryonic bradycardia, alone or corroborated with the size of the gestational sac [17]. Callen et al considers that identifying a DYSE of less than $3 \mathrm{~mm}$ in size, evidenced by embryos with CRL $>5 \mathrm{~mm}$ and without cardiac activity, even at an early stage, is defining for the diagnosis of embryonic demise. In the absence of the specific ultrasonographic signs of unfavorable evolutivity, listed above, identification of a DYSE $<3 \mathrm{~mm}$ at an early stage of pregnancy, respectively from 6 weeks of amenorrhea, could increase the accuracy of the diagnosis [15].

The data from our study confirmed that DYSE is an ultrasonographic parameter that indicates the risk of embryonic demise early. For an early and correct diagnosis, we considered that the correlation of DYSE with sFlt1 might be useful and could bring valuable information regarding the early development of the pregnancy [18].

It is noteworthy that a single serum determination of sFlt- 1 between 6 and 11 weeks of amenorrhea makes it possible to distinguish between a pregnancy with an unfavorable prognosis and a pregnancy with a favorable one [19]. Daponte et al had identified that an average value of sFlt-1 of $288.79 \mathrm{pg} / \mathrm{ml}$ is associated with embryonic demise and $1390.32 \mathrm{pg} / \mathrm{ml}$ is associated with viable pregnancies [9].

This study opens new perspectives in the assessment of the first-trimester pregnancy with potential reserved evolutivity by using the complementary data offered by ultrasonography and serological markers.

Most of the previous studies that have shown the ability of serum biomarkers to anticipate embryonic demise have been performed on cohorts of patients already experiencing symptoms of spontaneous abortion (bleeding and abdominal pain) $[20,21]$. However, evaluating a biomarker in this context is of limited clinical utility, knowing that at this stage, the most appropriate investigation is serial ultrasonographic examination [22]. The data from our study, unlike a previous study [23], highlights the importance of the parameters used for early identification of an unfavorable prognosis of pregnancy, when the embryo is still viable and the patients are asymptomatic. Also, more patients could be identified at 
an earlier stage of pregnancy and the ultrasound methodology and minimally invasive blood testing association ensured an accurate diagnosis for the patients enrolled in the study. On the other hand, the major limitation of this study consists of the fact that the study was conducted on a small number of patients, selected from the same region. Further larger studies are required to investigate the predictive efficiency of these markers. Also, the patients enrolled in the study passed through a stressful period once an abnormal DYSE was found until the final diagnosis. The patients were randomly selected and they were not investigated for certain specific associated pathologies, for example thrombophilia or antiphospholipidic syndrome, which may sometimes influence the prognosis of pregnancy.

\section{Conclusions}

A low level of sFlt-1 associated with a distance between the embryo and yolk sac of small dimensions, respectively $<3 \mathrm{~mm}$, correlates with an increased rate of non-viable embryos. This correlation between an ultrasound and a serum parameter is of great value and brings important information regarding the viability of first trimester pregnancies.

\section{Acknowledgements}

This paper was published under the project no 7690/15.04.2016 funded by "Iuliu Hatieganu" University of Medicine and Pharmacy, $2^{\text {nd }}$ Department of Obstetrics and Gynecology, Cluj-Napoca, Romania.

\section{References}

1. Elson J, Salim R, Tailor A, Banerjee S, Zosmer N, Jurkovic D. Prediction of early pregnancy viability in the absence of an ultrasonically detectable embryo. Ultrasound Obstet Gynecol 2003;21:57-61.

2. Azmanov DN, Milachich TV, Zaharieva BM, et al. Profile of chromosomal aberrations in different gestational age spontaneous abortions detected by comparative genomic hybridization. Eur J Obstet Gynecol Reprod Biol 2007;131:127-131.

3. Jauniaux E, Johns J, Burton GJ. The role of ultrasound imaging in diagnosing and investigating early pregnancy failure. Ultrasound Obstet Gynecol 2005;25:613-624.

4. Yegul NT, Filly RA. The expanded amnion sign: evidence of early embryonic death. J Ultrasound Med 2009;28:13311335.

5. Daponte A, Pournaras S, Zintzaras E, Kallitsaris A, Lialios $\mathrm{G}$, Maniatis AN, Messinis IE. The value of a single measurement of VEGF, glycodelin, progesterone, PAPP-A, HPL and LIF for differentiatingbetween ectopic and abnormal intrauterine pregnancy. Hum Reprod 2005;20:3163-3166.

6. Woods-Giscombé CL, Lobel M, Crandell JL. The Impact of Miscarriage and Parity on Patterns of Maternal Distress in Pregnancy. Res Nurs Health 2010;33:316-328.

7. Muttukrishna S, Suri S, Groome N, Jauniaux E. Relationships between TGFbeta proteins and oxygen concentrations inside the first trimester human gestational sac. PLoS One 2008;3:e2302.

8. Jacobs M, Nassar N, Roberts CL, Hadfield R, Morris JM, Ashton AW. Levels of soluble fms-like tyrosine kinase one in first trimester and outcomes of pregnancy: a systematic review. Reprod Biol Endocrinol 2011;9:77.

9. Daponte A1, Pournaras S, Polyzos NP, et al. Soluble FMSlike tyrosin kinase-1 (sFlt-1) and serum placental growth factor (PlGF) as biomarkers for ectopic pregnancy and missed abortion. J Clin Endocrinol Metab 2011;96:E1444-E1451.

10. Kaitu'u-Lino TJ, Whitehead CL, Ngian GL, Permezel M, Tong S. Serum concentrations of soluble Flt-1 are decreased among women with a viable fetus and no symptoms of miscarriage destined for pregnancy loss. PLoS One 2012;7:e32509.

11. Muttukrishna S, Swer M, Suri S, et al. Soluble Flt-1 and PlGF: new markers of early pregnancy loss. Plos One 2011;6:e18041.

12. Plaisier M, Rodrigues S, Willems F, Koolwijk P, van Hinsbergh VW, et al. Different degrees of vascularization and their relationship to the expression of vascular endothelial growth factor, placental growth factor, angiopoietins, and their receptors in first-trimester decidual tissues. Fertil Steril 2007; 88:176-187

13. Aziz S, Cho RC, Baker DB, Chhor C, Filly RA. Five- millimeter and smaller embryos without embryonic cardiac activity: outcomes in women with vaginal bleeding. J U1trasound Med 2008;27:1559-1561.

14. Hately W, Case J, Campbell S. Establishing the death of an embryo by ultrasound:report of a public inquiry with recommendations. Ultrasound Obstet Gynecol 1995;5:353357.

15. Laing FC, Frates MC, Benson CB. Ultrasound evaluation during the first trimester of pregnancy. In: Callen PW (ed). Ultrasonography in Obstetrics and Gynecology. 5th ed. Philadelphia, PA Saunders Elsevier 2008;181-224.

16. Brown DL, Emerson DS, Felker RE, Cartier MS, Smith WC. Diagnosis of early embryonic demise by endovaginal sonography. J Ultrasound Med 1990;9:631-636.

17. Salamanca A, Fernandez-Salmeron P, Beltran E, Mendoza N, Florido J, Mozas J. Early embryonic morphology sonographically assessed and its correlation with yolk sac i n missed abortion. Arch Gynecol Obstet 2013;287:139-142.

18. Ahmed A, Dunk C, Ahmad S, Khaliq A. Regulation of placental vascular endothelial growth factor (VEGF) and placenta growth factor (PIGF) and soluble Flt-1 by oxygen-a review. Placenta 2000;21 Suppl A:S16-S24.

19. Plaisier M, Dennert I, Rost E, Koolwijk P, van Hinsbergh VW, Helmerhorst FM. Decidual vascularization and the expression of angiogenic growth factors and proteases 
492 Carmen Elena Bucuri et al Yolk sac-embryo distance in correlation with soluble form of vascular endothelial growth factor levels

in first trimester spontaneous abortions. Hum Reprod 2009;24:185-197.

20. Duan L, Yan D, Zeng W, Yang X, Wei Q. Predictive power progesterone combined with beta human chorionic gonadotropin measurements in the outcome of threatened miscarriage. Arch Gynecol Obstet 2011;283:431-435.

21. Barnhart KT, Sammel MD, Rinaudo PF, Zhou L, Hummel AC, Guo W. Symptomatic patients with an early viable intrauterine pregnancy: HCG curves redefined. Obstet Gynecol 2004; 104:50-55.
22. Laing FC, Frates MC, Benson CB. Ultrasound evaluation during the first trimester of pregnancy. In: Callen PW (ed). Ultrasonography in Obstetrics and Gynecology 2008;181224.

23. Nagamatsu T, Fujii T, Kusumi M, et al. Cytotrophoblasts up-regulate soluble fms-like tyrosine kinase-1 expression under reduced oxygen: an implication for the placental vascular development and the pathophysiology of preeclampsia. Endocrinology 2004; 145:4838-4845. 